International Mathematical Forum, Vol. 8, 2013, no. 40, 1977 - 1984

HIKARI Ltd, www.m-hikari.com

http://dx.doi.org/10.12988/imf.2013.312231

\title{
Approximation of Pseudo-Inverse Operators by $g$-Frames
}

\author{
Mohammad Sadegh Asgari and Seyedeh Sara Karimizad \\ Department of Mathematics, Faculty of Science \\ Islamic Azad University, Central Tehran Branch, Tehran, Iran \\ moh.asgari@iauctb.ac.ir, msasgari@yahoo.com \\ s_karimizad@yahoo.com
}

Copyright (c) 2013 Mohammad Sadegh Asgari and Seyedeh Sara Karimizad. This is an open access article distributed under the Creative Commons Attribution License, which permits unrestricted use, distribution, and reproduction in any medium, provided the original work is properly cited.

\begin{abstract}
Let $T$ denote an operator on a Hilbert space $\mathcal{H}$ and let $\left\{\Lambda_{j}\right\}$ be a $g$-frame for the orthogonal complement of the kernel $N_{T}$. We construct a sequence of operators $\left\{\phi_{n}\right\}$ of the form $\phi_{n}()=.\sum_{j=1}^{n} g_{j}^{n}(.) \Lambda_{j}$ which converges to the psuedoinverse $T^{\dagger}$ of $T$ in the strong operator topology as $n \rightarrow \infty$. The operators $\left\{\phi_{n}\right\}$ can be found using finite-dimensional methods. We also prove an adaptive iterative version of the result.
\end{abstract}

Mathematics Subject Classification: Primary 42C99; Secondary 46B99, 46C99

Keywords: $g$-frames, approximate frames, pseudo-inverse

\section{Introduction}

Let $\mathcal{H}, \mathcal{K}$ be two separable Hilbert spaces and $\left\{W_{j}\right\}_{j \in J}$ be a sequence of closed subspaces of $\mathcal{K}$, where $J$ is a subset of $\mathbb{Z}$. Let $\mathcal{B}\left(\mathcal{H}, W_{j}\right)$ be the collection of all bounded linear operators from $\mathcal{H}$ into $W_{j}$. For each sequence $\left\{W_{j}\right\}_{j \in J}$, we define the space $\left(\sum_{j \in J} \oplus W_{j}\right)_{\ell^{2}}$ by

$$
\left(\sum_{j \in J} \oplus W_{j}\right)_{\ell^{2}}=\left\{\left\{g_{j}\right\}_{j \in J} \mid g_{j} \in W_{j} \text { and } \sum_{j \in J}\left\|g_{j}\right\|^{2}<\infty\right\} .
$$

With the inner product defined by

$$
\left\langle\left\{f_{j}\right\},\left\{g_{j}\right\}\right\rangle=\sum_{j \in J}\left\langle f_{j}, g_{j}\right\rangle .
$$

It is clear that $\left(\sum_{j \in J} \oplus W_{j}\right)_{\ell^{2}}$ is a Hilbert space. 
Definition 1.1. Let $\Lambda_{j} \in \mathcal{B}\left(\mathcal{H}, W_{j}\right)$ for all $j \in J$. A family $\Lambda=\left\{\Lambda_{j}\right\}_{j \in J}$ is called a generalized frame or simply a $g$-frame for $\mathcal{H}$ with respect to $\left\{W_{j}\right\}_{j \in J}$ if there exist constants $0<A \leq B<\infty$ such that

$$
A\|f\|^{2} \leq \sum_{j \in J}\left\|\Lambda_{j} f\right\|^{2} \leq B\|f\|^{2} \quad \forall f \in \mathcal{H} .
$$

The constants $A$ and $B$ are called $g$-frame bounds. The synthesis operator of $\Lambda$ given by

$$
T_{\Lambda}:\left(\sum_{j \in J} \oplus W_{j}\right)_{\ell^{2}} \rightarrow \mathcal{H} \quad T_{\Lambda}\left(\left\{g_{j}\right\}_{j \in J}\right)=\sum_{j \in J} \Lambda_{j}^{*} g_{j}
$$

The adjoint operator of $T_{\Lambda}$, which is called the analysis operator also obtain as follows

$$
T_{\Lambda}^{*}: \mathcal{H} \rightarrow\left(\sum_{j \in J} \oplus W_{j}\right)_{\ell^{2}} \quad T_{\Lambda}^{*} f=\left\{\Lambda_{j} f\right\}_{j \in J} .
$$

By composing $T_{\Lambda}$ with its adjoint $T_{\Lambda}^{*}$, we obtain the generalized frame operator

$$
S_{\Lambda}: \mathcal{H} \rightarrow \mathcal{H}, \quad S_{\Lambda} f=T_{\Lambda}, \quad T_{\Lambda}^{*} f=\sum_{j \in J} \Lambda_{j}^{*} \Lambda_{j} f
$$

which is a bounded, self-adjoint, positive and invertible operator and $C I_{\mathcal{H}} \leq S_{\Lambda} \leq D I_{\mathcal{H}}$. We call the operators $T$ and $T^{*}$, synthesis and analysis operators, respectively. A $g$-frame for a subspace yields a representation of the orthogonal projection onto the subspace. Given a sequence $\left\{\Lambda_{j}\right\}_{j \in J}$, let $P_{J}$ denote the orthogonal projection onto $\overline{\operatorname{span}}\left\{\Lambda_{j}^{*}\left(W_{j}\right)\right\}_{j \in J}$. Let $\left\{\Lambda_{j} \in \mathcal{B}\left(\mathcal{H}, W_{j}\right): j \in J\right\}$ be a $g$-Bessel sequence for $\mathcal{H}$. The operator

$$
S: \mathcal{H} \longrightarrow \mathcal{H}, \quad S f=\sum_{j \in J} \Lambda_{j}^{*} \Lambda_{j} f
$$

is a positive and bounded operator.

A simple computation shows that

$$
\langle S f, f\rangle=\left\langle\sum_{j \in J} \Lambda_{j}^{*} \Lambda_{j} f, f\right\rangle=\sum_{j \in J}\left\langle\Lambda_{j} f, \Lambda_{j} f\right\rangle=\sum_{j \in J}\left\|\Lambda_{j} f\right\|^{2} \quad \forall f \in \mathcal{H} .
$$

Therefore

$$
A\langle f, f\rangle \leq\langle S f, f\rangle \leq B\langle f, f\rangle
$$

i.e.,

$$
A I \leq S \leq B I
$$

This implies that $S$ is an invertible operator if and only if $\left\{\Lambda_{j} \in \mathcal{B}\left(\mathcal{H}, W_{j}\right): j \in J\right\}$ is a $g$-frame for $\mathcal{H}$. For more details about the theory and applications of generalized frames we refer the readers to $[4,5]$. 


\section{Linear approximation of pseudo-inverse operators}

Let $\mathcal{H}, \mathcal{K}$ be two Hilbert spaces and $\mathcal{B}(\mathcal{H}, \mathcal{K})$ the set of bounded linear operators from $\mathcal{H}$ into $\mathcal{K}$. The range and the null space of $T \in \mathcal{B}(\mathcal{H}, \mathcal{K})$ are denoted by $R_{T}$ and $N_{T}$ ,respectively. Suppose that the operator $T \in \mathcal{B}(\mathcal{H}, \mathcal{K})$ has a closed range. Then there exists a unique bounded operator $T^{\dagger}: \mathcal{K} \longrightarrow \mathcal{H}$ satisfying:

$$
T T^{\dagger} T=T, T^{\dagger} T T^{\dagger}=T^{\dagger},\left(T^{\dagger} T\right)^{*}=T^{\dagger} T,\left(T T^{\dagger}\right)^{*}=T T^{\dagger}
$$

The operator $T^{\dagger}$ is called the pseudo-inverse operator of $T$. If $T$ is a bounded invertible operator, then $T^{\dagger}=T^{-1}$. Alternatively, $T^{\dagger}$ can be characterized as the unique linear operator from $\mathcal{H}$ to $\mathcal{H}$ for which

$$
N_{T}^{\dagger}=R_{T}^{\perp} \quad, R_{T}^{\dagger}=N_{T}^{\perp} \quad, T T^{\dagger} f=f \quad \forall f \in R_{T} .
$$

It is well known that $T T^{\dagger}$ is the orthogonal projection of $\mathcal{H}$ onto $R_{T}$ and that $T^{\dagger} T$ is the orthogonal projection of $\mathcal{H}$ onto $N_{T}^{\perp}$. Let $P_{R_{T}}$ denote the orthogonal projection of $\mathcal{H}$ onto $R_{T}$ and observe that for arbitrary $f \in \mathcal{H}$ we have

$$
\left(I-P_{R_{T}}\right) f \in R_{T}^{\perp}=N_{T} \dagger
$$

Therefore

$$
T^{\dagger} f=T^{\dagger} P_{R_{T}} f+T^{\dagger}\left(I-P_{R_{T}}\right) f=T^{\dagger} P_{R_{T}} f .
$$

The purpose of this note is to present a method for approximation of $T^{\dagger}$. Let $T$ : $\mathcal{H} \longrightarrow \mathcal{H}$ be a bounded linear operator with closed range $R_{T}$. let $\left\{\Lambda_{i}\right\}_{i \in I}$ be a $g$ frame for the subspace $N_{T}^{\perp}$ with respect to $\left\{W_{i}\right\}_{i \in I}$. For each finite subset $J \subseteq I$, let $\mathcal{H}_{J}=\overline{\operatorname{span}}\left\{\Lambda_{j}^{*}\left(W_{j}\right)\right\}_{j \in J}$. Then $\left\{\Lambda_{j}\right\}_{j \in J}$ is a $g$-frame for $\mathcal{H}_{J}$ with respect to $\left\{W_{j}\right\}_{j \in J}$ with $g$-frame operator given by

$$
S_{J}: \mathcal{H}_{J} \longrightarrow \mathcal{H}_{J} \quad S_{J} f=\sum_{j \in J} \Lambda_{j}^{*} \Lambda_{j} f
$$

Also, $\left\{\Lambda_{j} T^{*}\right\}_{j \in J}$ is a $g$-frame for $T\left(\mathcal{H}_{J}\right)$ with respect to $\left\{W_{j}\right\}_{j \in J}$ with $g$-frame operator given by

$$
V_{J}: \overline{T\left(\mathcal{H}_{J}\right)} \longrightarrow \overline{T\left(\mathcal{H}_{J}\right)} \quad V_{J} f=\sum_{j \in J} T \Lambda_{j}^{*} \Lambda_{j} T^{*} f \quad \forall f \in \overline{T\left(\mathcal{H}_{J}\right)}
$$

where $V_{J}$ is a invertible, bounded linear operator onto $T\left(\mathcal{H}_{J}\right)$. When in the following we write $V_{J}^{-1}$, it is understood that we invert $V_{J}$ as an operator from $\overline{T\left(\mathcal{H}_{J}\right)}$ onto $\overline{T\left(\mathcal{H}_{J}\right)}$. Also for all $f \in \overline{T\left(\mathcal{H}_{J}\right)}$ we have

$$
V_{J} f=\sum_{j \in J} T \Lambda_{j}^{*} \Lambda_{j} T^{*} f=T\left(\sum_{j \in J} \Lambda_{j}^{*} \Lambda_{j}\right)\left(T^{*} f\right)=T S_{J} P_{J} T^{*} f
$$

or

$$
V_{J} f=\sum_{j \in J} T \Lambda_{j}^{*} \Lambda_{j} T^{*} f=T\left(\sum_{j \in J} \Lambda_{j}^{*} \Lambda_{j}\right)\left(T^{*} f\right)=T P_{J} S_{J} T^{*} f
$$


Hence for all $f \in \overline{T\left(\mathcal{H}_{J}\right)}$

$$
V_{J} f=T P_{J} S_{J} T^{*} f=T S_{J} P_{J} T^{*} f .
$$

Lemma 2.1. Let $\left\{\Lambda_{j}\right\}_{j \in J}$ be a $g$-frame sequence for $\mathcal{H}$ with respect to $\left\{w_{j}\right\}_{j \in J}$. Then the orthogonal projection onto $H_{0}=\overline{\operatorname{span}}\left\{\Lambda_{j}^{*}\left(w_{j}\right)\right\}_{j \in J}$ is given by

$$
P_{H_{0}}=\sum_{j \in J} S_{\Lambda}^{-1} \Lambda_{j}^{*} \Lambda_{j} f \quad \forall f \in \mathcal{H}
$$

Proof. By assumption we have $S^{-1}\left(H_{0}\right)=H_{0}$. Hence for all $g \in \mathcal{H}$ we have

$$
\begin{aligned}
\left\langle\sum_{j \in J} S_{\Lambda}^{-1} \Lambda_{j}^{*} \Lambda_{j} f, g\right\rangle & =\sum_{j \in J}\left\langle S_{\Lambda}^{-1} \Lambda_{j}^{*} \Lambda_{j} f, g\right\rangle=\sum_{j \in J}\left\langle P_{H_{0}} S_{\Lambda}^{-1} \Lambda_{j}^{*} \Lambda_{j} f, g\right\rangle=\left\langle f, \sum_{j \in J} \Lambda^{*} j \Lambda_{j} S_{\Lambda}^{-1} P_{H_{0}} g\right\rangle \\
& =\left\langle f, P_{H_{0}} g\right\rangle=\left\langle P_{H_{0}} f, g\right\rangle
\end{aligned}
$$

Hence $P_{H_{0}} f=\sum_{j \in J} S_{\Lambda}^{-1} \Lambda_{j}^{*} \Lambda_{j} f$.

Lemma 2.2. Let $f \in \mathcal{H}$ be and $I \subseteq J$ be finite.Then

$$
i n f_{\phi \in \mathcal{H}_{I}}\|f-T \phi\|=\|f-T \psi\|
$$

where $\psi$ is defined by

$$
\psi=\sum_{j \in I} \Lambda_{j}^{*} \Lambda_{j} T^{*} V_{I}^{-1} f \quad \text { for all } \quad f \in \mathcal{H}
$$

Proof. Let $f \in \mathcal{H}$ be arbitary and $W \subseteq \mathcal{H}$. We have $\left\|f-P_{W} f\right\|=\inf _{h \in W}\|f-h\|$ where $P_{W}: \mathcal{H} \longrightarrow W$ is orthogonal projection. Then

$$
\inf _{\phi \in \mathcal{H}_{I}}\|f-T \phi\|=\inf _{h \in T \mathcal{H}_{I}}\|f-h\|=\left\|f-P_{T \mathcal{H}_{I}} f\right\| .
$$

In addition

$$
P_{T \mathcal{H}_{I}} f=\sum_{j \in I} T \Lambda_{j}^{*} \Lambda_{j} T^{*} \Lambda_{j}^{-1} f
$$

Hence

$$
P_{T \mathcal{H}_{I}} f=\sum_{j \in I} T \Lambda_{j}^{*} \Lambda_{j} T^{*} V_{j}^{-1} f=T\left(\sum_{j \in I} \Lambda^{*} j \Lambda_{j} T^{*} V_{j}^{-1} f\right) .
$$

Put

$$
\psi=\sum_{j \in I} \Lambda_{j}^{*} \Lambda_{j} T^{*} V_{j}^{-1} f=T\left(\sum_{j \in I} \Lambda_{j}^{*} \Lambda_{j} T^{*} V_{j}^{-1} f\right)
$$

Therefore we obtain

$$
\inf _{\phi \in \mathcal{H}_{I}}\|f-T \phi\|=\inf _{h \in T \mathcal{H}_{I}}\|f-h\|=\left\|f-P_{T \mathcal{H}_{I}} f\right\|=\|f-T \psi\| .
$$


Lemma 2.2 leads to a method for approximation of $T^{\dagger} f$. Let $\left\{I_{n}\right\}_{n \in \mathbb{N}}$ be a family of finite subsets of $I$ such that $I_{1} \subseteq I_{2} \ldots \subseteq I_{n} \nearrow I$. Abusing the notation, we will write $\mathcal{H}_{n}, S_{n}, V_{n}, P_{n}$ instead of $\mathcal{H}_{I_{n}}, S_{I_{n}}, V_{I_{n}}, P_{I_{n}}$. The following lemma states that for $f \in R_{T}$ we can make $\inf _{\phi \in \mathcal{H}_{n}}\|f-T \phi\|$ arbitrarily small by choosing $n$ large enough.

Lemma 2.3. Let $f \in R_{T}$ be, then

$$
\lim _{n \rightarrow \infty} \inf _{\phi} \in \mathcal{H}_{n}\|f-T \phi\|=0 .
$$

Proof. By lemma (2.2) we have

$$
\lim _{n \rightarrow \infty} \inf _{\phi} \in \mathcal{H}_{n}\|f-T \phi\|=\lim _{n \rightarrow \infty}\left\|f-P_{T \mathcal{H}_{n}} f\right\|
$$

we show given $f \in \mathcal{H}$,

$$
\lim _{n \rightarrow \infty} P_{T \mathcal{H}_{n}} f=f
$$

Since $I_{1} \subseteq I_{2} \subseteq \ldots \subseteq I_{n} \nearrow I$ and $\left\{\Lambda_{j}\right\}_{j \in J}$ is a $g$-frame for $N_{T}^{\perp}$ with respect to $\left\{W_{j}\right\}_{j \in J}$ therefore

$$
N_{T}^{\perp}=\overline{\operatorname{span}}\left\{\Lambda_{j}^{*}\left(W_{j}\right)\right\}
$$

and

$$
\mathcal{H}_{1} \subseteq \mathcal{H}_{2} \subseteq \ldots \subseteq \mathcal{H}_{n} \nearrow N_{T}^{\perp}
$$

Then

$$
N_{T}^{\perp}=\overline{\operatorname{span}}\left\{\mathcal{H}_{n}\right\}
$$

and

$$
T N_{T}^{\perp}=\overline{\operatorname{span}}\left\{T \mathcal{H}_{n}\right\}_{n \in \mathbb{N}}
$$

On the other hand

$$
T(\mathcal{H})=T\left(N_{T} \oplus N_{T}^{\perp}\right)=T\left(N_{T}^{\perp}\right)=\overline{\operatorname{span}}\left\{T\left(\mathcal{H}_{n}\right)\right\}_{n \in \mathbb{N}}
$$

Since

$$
T\left(\mathcal{H}_{1}\right) \subseteq T\left(\mathcal{H}_{2}\right) \subseteq \ldots \subseteq T\left(\mathcal{H}_{n}\right) \nearrow T(\mathcal{H})
$$

Therefore given $f \in \mathcal{H}$

$$
\lim _{n \rightarrow \infty} P_{T\left(\mathcal{H}_{n}\right)} P_{T(\mathcal{H})} f=P_{T(\mathcal{H})} f \quad \text { and } \quad \lim _{n \rightarrow \infty} P_{T\left(\mathcal{H}_{n}\right)} f=f \quad \text { forall } \quad f \in R_{T}
$$

Then

$$
\lim _{n \rightarrow \infty} i n f_{\phi \in \mathcal{H}_{n}}\|f-T \phi\|=\lim _{n \rightarrow \infty}\left\|f-P_{T \mathcal{H}_{n}} f\right\|=0 .
$$


The next theorem shows how we can obtain a family of operators $\left\{\phi_{n}\right\}_{n \in \mathbb{N}}$ that converges to $T^{\dagger}$ in the strong operator topology.

Lemma 2.4. For $n \in \mathbb{N}$, define $\phi_{n}: \mathcal{H} \longrightarrow \mathcal{H}$ by

$$
\phi_{n} f=\sum_{j \in J_{n}} \Lambda_{j}^{*} \Lambda_{j} T^{*} V_{n}^{-1} f
$$

then

$$
\lim _{n \rightarrow \infty} \phi_{n} f=T^{\dagger} f .
$$

Proof. Let $f \in \mathcal{H}$. We have $T \phi_{n} f=\sum_{j \in J_{n}} T \Lambda_{j}^{*} \Lambda_{j} T^{*} V_{n}^{-1} f=P_{T(\mathcal{H} n)} f$ as $n \rightarrow \infty$.

Then

$$
\lim _{n \rightarrow \infty} T \phi_{n} f=\lim _{n \rightarrow \infty} P_{T(\mathcal{H} n)} f=P_{T(\mathcal{H})} f=T T^{\dagger} f .
$$

Since

$$
\overline{\operatorname{span}}\left\{\Lambda_{j}^{*}\left(W_{j}\right)\right\}_{j \in J}=N_{T}^{\perp}
$$

and given $n \in \mathbb{N}$, we have $\phi_{n} f \in N_{T}^{\perp}$. Then

$$
T^{\dagger} T \phi_{n} f=\phi_{n} f .
$$

It follows that

$$
\lim _{n \rightarrow \infty} \phi_{n} f=\lim T^{\dagger} T T^{\dagger} f=T^{\dagger} f .
$$

\section{Nonlinear iterative approximation of $T^{\dagger} f$}

In the previous section, the index sets $\left\{I_{n}\right\}_{n \in \mathbb{N}}$ were fixed independentlyof $f$. As a consequence, we obtained a family of operators $\left\{\phi_{n}\right\}$ convergingto $T^{\dagger}$ in the strong operator topology. We now describe an elementdependent method for approximation of $T^{\dagger} f$. This means that we fix $f \in \mathcal{H}$ and that the choice of $\left\{I_{n}\right\}_{n \in \mathbb{N}}$ depends on $f$. The advantage is that the choice of In at the nth step of the approximation might fit $f$ better, but the disadvantage is that the method becomes nonlinear. This method is inspired by various versions of matching pursuit algorithms; cf. $[1,2,3]$. Corresponding to an index set $I_{n}$, we use the notation $\mathcal{H}_{n}, S_{n}, V_{n}$ as in Section 2. First, fix $f \in \mathcal{H}$ and let $\varepsilon>0$ be given. Choose the set $I_{1}$ such that

$$
\left\|P_{R_{T}} f-P_{T \mathcal{H}_{1}} P_{R_{T}} f\right\| \leq \varepsilon
$$

Write $P_{R_{T}} f=P_{T \mathcal{H}_{1}} P_{R_{T}} f+R_{1}$ and observe that

$$
\left\|P_{R_{T}} f\right\|^{2}=\left\|P_{T \mathcal{H}_{1}} P_{R_{T}} f\right\|^{2}+\left\|R_{1}\right\|^{2}
$$


Now choose $I_{2}$ such that $\left\|R_{1}-P_{T \mathcal{H}_{2}} R_{1}\right\| \leq \varepsilon$. Write $R_{1}=P_{T \mathcal{H}_{2}} R_{1}+R_{2}$ and observe that

$$
\left\|R_{2}\right\|=\left\|R_{1}-P_{T \mathcal{H}_{2}} R_{1}\right\| \leq \varepsilon
$$

Thus

$$
\left\|P_{R_{T}} f-\left(P_{T \mathcal{H}_{1}} P_{R_{T}} f+P_{T \mathcal{H}_{2}} R_{1}\right)\right\|=\left\|R_{2}\right\| \leq \varepsilon .
$$

In general, after constructing $R_{n}$, $\operatorname{choose} I_{n+1}$ such that

$$
\left\|R_{n}-P_{T H_{n+1}} R_{n}\right\| \leq \varepsilon\left(2^{-n-1}\right) .
$$

Write $R_{n}=P_{T \mathcal{H}_{n+1}} R_{n}+R_{n+1}$ and observe that $\left\|R_{n+1}\right\| \leq \varepsilon\left(2^{-n-1}\right)$. Thus, with $R_{0}=$ $P_{R_{T}} f$ we have

$$
\left\|P_{R_{T}} f\right\|^{2}=\sum_{k=0}^{n}\left\|P_{T \mathcal{H}_{k+1}} R_{k}\right\|^{2}+\left\|R_{n+1}\right\|^{2} .
$$

Since $\left\{\Lambda_{i} T^{*}\right\}_{i \in I_{k+1}}$ is a $g$-frame for $T \mathcal{H}_{k+1}$ and the corresponding $g$-frame operatoris $V_{k+1}$, we have by Lemma 2.1 that

$$
P_{T \mathcal{H}_{k+1}} R_{k}=\sum_{j \in J_{k+1}} T \Lambda_{j}^{*} \Lambda_{j} T^{*} V_{k+1}^{-1} R_{k}
$$

Put $g_{k}=\sum_{j \in J_{k+1}} \Lambda_{j}^{*} \Lambda_{j} T^{*} V_{k+1}^{-1} R_{k}$ then $P_{T \mathcal{H}_{k+1}} R_{k}=T g_{k}$. We obtain

$$
\left\|P_{R_{T}} f-T \sum_{k=0}^{n} g_{k}\right\|<\varepsilon\left(2^{-n-1}\right)
$$

The iterative approximation of $P_{R_{T}} f$ leads to the following result on approximation of $T^{\dagger} f$.

Lemma 3.1. Let $f \in \mathcal{H}$ be and construct $\left\{g_{k}\right\}_{k=0}^{\infty}$ as above. Then

$$
\left\|T^{\dagger} f-\sum_{k=0}^{n} g_{k}\right\| \leq \varepsilon\left(2^{-n-1}\right)\left\|T^{\dagger}\right\|
$$

Proof. First observe that for all $k \in \mathbb{N}$ we have

$$
g_{k} \in \overline{\operatorname{span}}\left\{\Lambda_{j}^{*}\left(W_{j}\right)\right\}_{j \in J}=N_{T}^{\perp}
$$

also $T^{\dagger} f \in R_{T^{\dagger}}=N_{T}^{\perp}$, and since $T^{\dagger} T: \mathcal{H} \longrightarrow N_{T}^{\perp}$ is the orthogonal projection onto $N_{T}^{\perp}$. We obtain

$$
\begin{aligned}
\left\|T^{\dagger} f-\sum_{k=0}^{n} g_{k}\right\| & =\left\|T^{\dagger} T T^{\dagger} f-T^{\dagger} T\left(\sum_{k=0}^{n} g_{k}\right)\right\| \leq\left\|T^{\dagger}\right\|\left\|T T^{\dagger} f-T \sum_{k=0}^{n} g_{k}\right\| \\
& =\left\|T^{\dagger}\right\|\left\|P_{R_{T}} f-T \sum_{k=0}^{n} g_{k}\right\|<\varepsilon\left(2^{-n-1}\right)\left\|T^{\dagger}\right\| .
\end{aligned}
$$


Let $P$ denote the orthogonal projection onto span $\left\{\Lambda_{j} T^{*}\right\}_{j \in k+1}$. Then

$$
\|f-P f\| \leq\left\|f-T \sum_{k=0}^{n} g_{k}\right\| .
$$

By writing $P f=T g, g$ might approximate $T^{\dagger} f$ even better than $\sum_{k=0}^{n} g_{k}$. However, for large index sets, calculation of $g$ becomes more involved because of the need to invert the frame operator corresponding to $\left\{\Lambda_{j} T^{*}\right\}_{j \in k+1}$.

The motivation behind the iterative method is to split the inversion into successive inver-

sions of smaller matrices. However, in the worst case the index set $I_{n}$ may have a lot of overlap with $I_{1}, I_{2}, \ldots, I_{n-1}$ (or even include those sets) and then the iterative method is not appropriate.

\section{References}

[1] F. J. Beutler and W. L. Root, The operator pseudo-inverse in control and system identifications, in "Generalized Inverses and Applications" (M. Zuhair Nashed, Ed.), Academic Press, New York, 1976.

[2] P. G. Casazza and O. Christensen, Approximation of the inverse frame operator and applications to Weyl-Heisenberg frames, J. Approx. Theory 103, No. 2 (2000), 338356.

[3] P. G. Casazza and O. Christensen, Riesz frames and approximation of the frame coefficients. Appr. Theory and Appl. 14 no. 2(1998). Appl. Comput. Harmon. Anal. 31(1) (2011) 1-25.

[4] W. Sun, G-frames and G-Riesz bases, J. Math. Anal. Appl. (2006), 322, 437-452.

[5] Zhu, Y. C, Characterizations of g-frames and g-Riesz bases in Hilbert spaces. Acta Mathematica Sinica, English Series, 24(10), 1727-1736 (2008)

Received: December 1, 2013 